\title{
Numerical analysis and optimization of the flow field in a new photocatalytic reactor for air treatment
}

\author{
Xiaoli Zhou, Li Zhang*, Lihua Dong \\ College of Ocean Sciences and Engineering,Shanghai Maritime University,Shanghai,201306,China \\ *zhangli@shmtu.edu.cn
}

Key words: photocatalytic reactor, numerical simulation, structure optimization

\begin{abstract}
A novel fixed bed photocatalytic reactor with tilted photocatalyst-coated deflectors was designed and a numerical simulation for evaluation of its photocatalytic performance by ANSYS FLUENT 15.0 was developed. The results showed that the convective mass transfer was effective near the deflectors. The air flow velocities were pretty fast with a maximum value of $0.55 \mathrm{~m} / \mathrm{s}$ and had larger longitudinal velocity which could enhance laminar mass transfer, near the front face where the reaction took place. While behind the deflector, the air flow even keep still and the pressure loss was $0.18 \mathrm{~Pa}$. Optimized construction of the photocatalytic reactor was designed and simulated for effects verification. The results showed that the velocity distributed uniformly near the inlet and outlet and the total pressure loss decreased to 0.09Pa.
\end{abstract}

\section{Introduction}

During the last decades much attention has been paid to the Photocatalytic reactions, because of its high degradation efficiency to organic pollutents, low energy consumption, no secondary pollution and mild reaction conditions [1,2].So far, most of the researches focused on the photocatalytic reaction kinetics and photocatalysts modification. However, the photocatalytic reaction occurs only at the photocatalyst surface irradiated with light [3], so the contaminant removal effectiveness depends on the mass transfer effect largely, which is determined by the structure of the reactor. To meet the plotocatalytic reaction's requirements of large reaction area, stable performance and high effectiveness, the structure of reactor needs further study.

At present, a novel photocatalytic reactor, which provided large area for reaction and high convective mass transfer efficiency, was designed. A numerical simulation for effects verification was developed and the structure of the reactor was optimized further.

\section{Prototype design}

It was as known, the main technical parameters of photocatalytic reactor are converctive mass transfer efficiency, reaction area and irradiance. In addition, easy installation and maintenance of the apparatus, economic usefulness of the set of equipment should be considered as well [4-7].

Reactor type selection. Loading-type reactor, which contains fluidized bed and fixed bed, is easy to maintain and recycle, suitable for industrial application. In a fluidized bed, photocatalysts are supported by spherical carrier, and move around as the air flow, e.g. Lynette A. Dibble etc [8] designed a small size fluidized bed for air purification of trichloroethylene. During the mixing process, the carriers would collide with each other inevitably. As a result, the photocatalyst coating would likely be worn away, and reduce the service life time. On the other hand, the blocking with each other would reduce the irradiance. In this work, the fixed bed type reactor is chosen.

Structure design. A reactor with $200 \mathrm{~mm}$ wide, $250 \mathrm{~mm}$ high and $500 \mathrm{~mm}$ long, as shown in Fig. 1 was designed. Successive alternating banks of UV lamps and photocatalyst-coated deflectors were mounted in a rectangular duct. The photocatalyst-coated deflectors were prepared by titanium plate as support and sol-gel process photocatalyst film as coating [9]. 


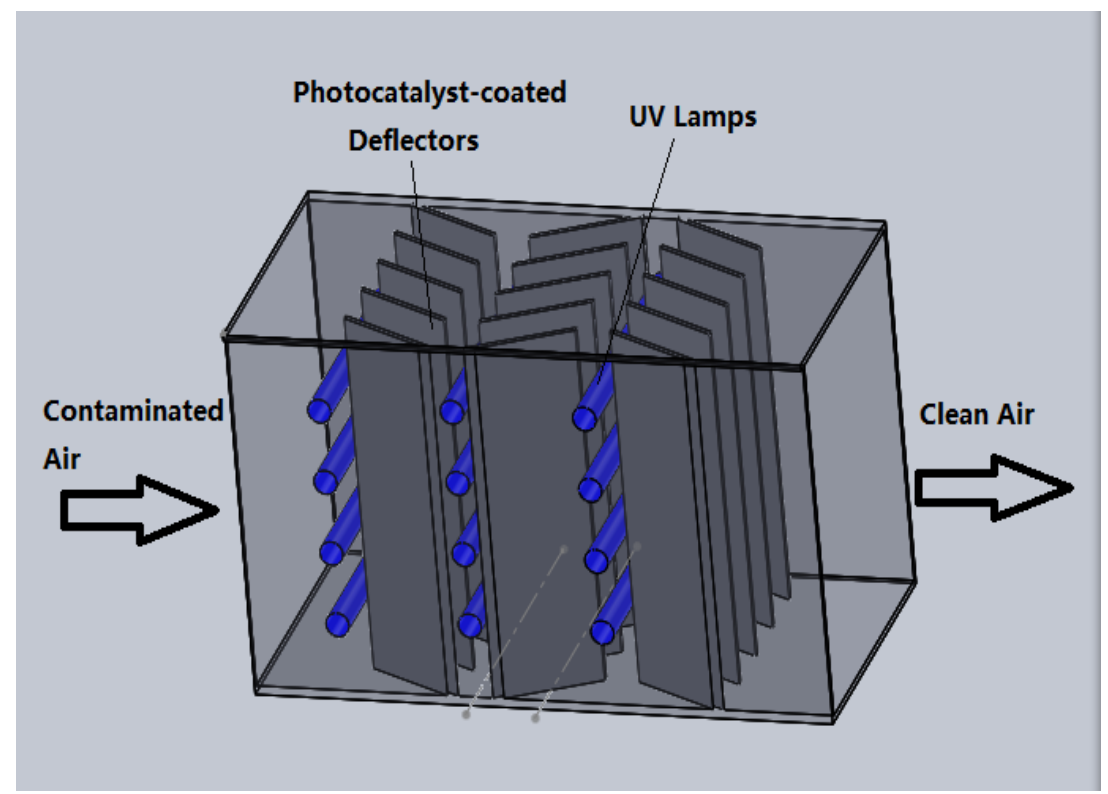

Fig.1 Schematic diagram of the photocatalytic reactor

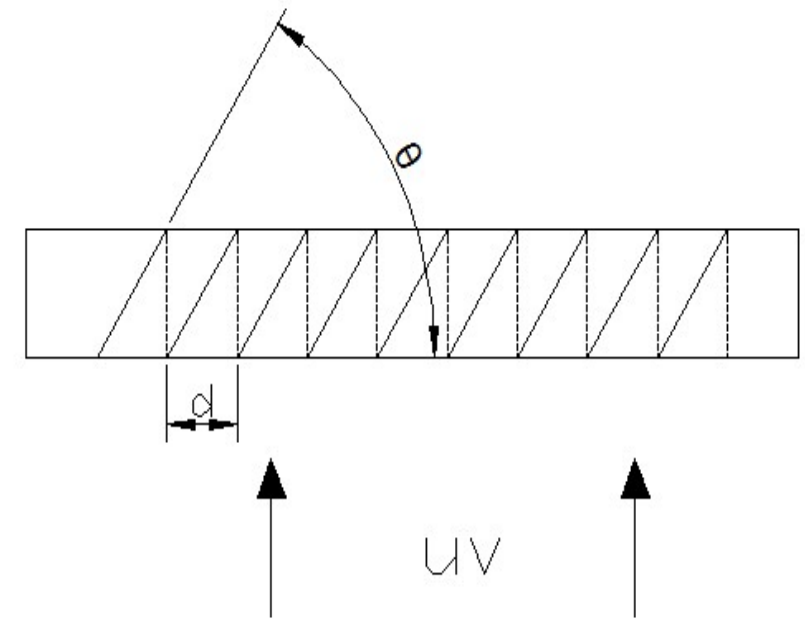

Fig.2 Geometric relationship diagram of a periodic segment

The angle of the inclination $(\theta)$, as shown in Fig.2, related directly to the number of deflectors $(n)$ and total reaction area $(A)$.

$$
\begin{aligned}
& \mathrm{n}=\frac{\mathrm{D}}{\mathrm{d}^{*}} \\
& \mathrm{~d}=\mathrm{l} * \cos (\theta) ; \\
& \mathrm{A}=\mathrm{n} * \mathrm{l} * \mathrm{H}
\end{aligned}
$$

Where $D, H, l$ represent the width, height of the reactor and the width of a deflector, respectively. With the increasing of $\theta$ the reaction area will increase. While, the number of deflectors $(n)$ increase would lead to construction complication. So in our design, $\theta$ was set to $60^{\circ}$, and $n$ was set to 8 .

\section{Numerical analysis}

The governing equation. For simplicity, the follow assumptions are made: (1)the reactants are single-phase gas, ignore the influence of the dust; (2)the density of the gas is constant; (3)the fluid is stationary flow; (4)ignore the influences of gravity and the lamps' structure. For these assumptions, the fluid field can be described by the following equation:

Mass continuity equation:

$$
\nabla \cdot \rho \vec{V}=0
$$

Where $\rho$ is the density of the air; is the velocity vector of the air. 
Momentum equation:

$p \vec{V} \cdot \nabla \vec{V}=-\nabla P+\nabla \cdot(\mu \nabla \vec{V})+\vec{F}$

Where $\mu$ is the dynamic viscosity and $P$ is the pressure.

$k-\varepsilon$ equation:

$$
\begin{aligned}
& \frac{\partial(\rho \mathrm{k})}{\partial \mathrm{t}}+\nabla \cdot(\rho \vec{V} \mathrm{k})=-\frac{2}{3} \rho \mathrm{k} \nabla \cdot \vec{V}+\tau \cdot \nabla \vec{V}+\nabla \cdot\left[\left(\frac{\mu_{z}}{\sigma_{k}}\right) \nabla \mathrm{k}\right]-\rho \varepsilon \\
& \frac{\partial(\rho \varepsilon)}{\partial \mathrm{t}}+\nabla \cdot(\rho \vec{V} \varepsilon)=-\left(\frac{2}{3} C_{1}-C_{a}\right) \cdot \rho \varepsilon \nabla \cdot \vec{V}+\nabla \cdot\left[\left(\frac{\mu_{g}}{\sigma_{z}}\right) \nabla \varepsilon\right]+\frac{\varepsilon}{k}\left(C_{1} \tau \cdot \nabla \vec{V}-C_{2} \rho \varepsilon\right) \\
& \mu_{\varepsilon}=\mu+C_{\mu} \rho^{2} / \varepsilon
\end{aligned}
$$

Where $k$ is the turbulent kinetic energy, $\varepsilon$ is the turbulent dissipation rate and $\mathrm{C}_{1}, \mathrm{C}_{2}, \mathrm{C}_{3}$ are constants in the turbulence model.

Numerical simulation. The two-dimensional mesh formed by ANSYS ICEM, was more condense near the deflector. The CFD program, ANSYS FLUENT 15.0, was used to simulate the flow field. Set air as the simulation fluid and the temperature as $22^{\circ} \mathrm{C}$. Boundary velocity-inlet was set as 2.2 $\mathrm{m} / \mathrm{s}$, pressure-outlet as $0 \mathrm{~Pa}$, and other boundary was as wall. After 240 times of iterative computation, the velocity field and pressure field were shown as Fig.3 and Fig.4 .

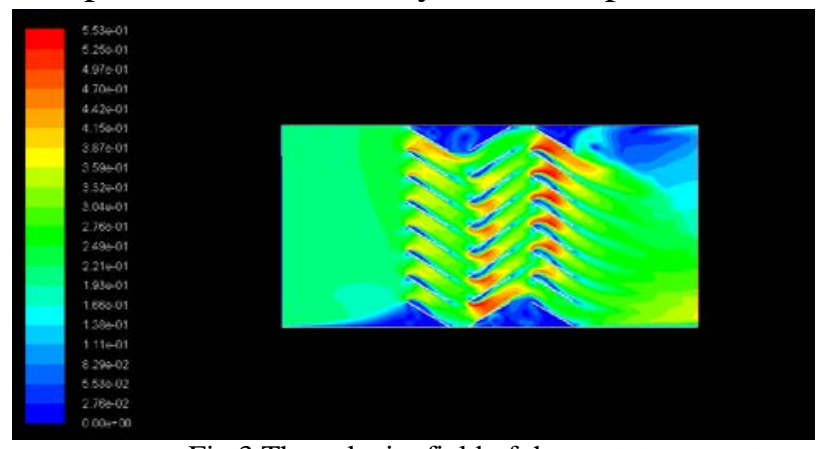

Fig.3 The velocity field of the prototype reactor

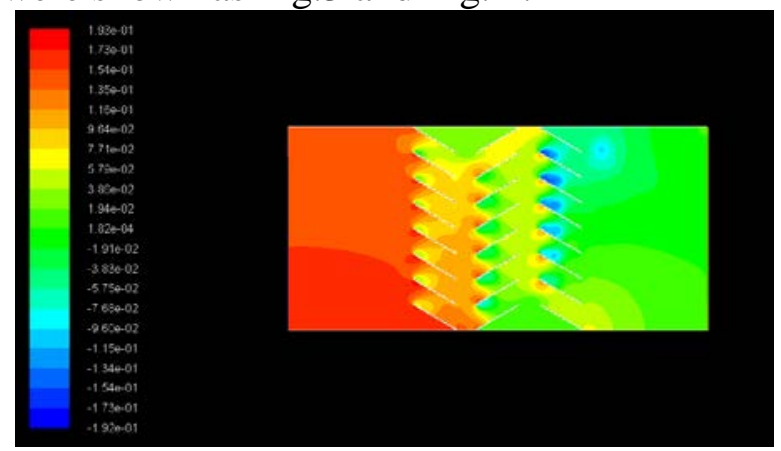

Fig.4 The pressure field of the prototype reactor

It was found that the air velocity distribution at the inlet was not uniform when guide by the deflectors. The air flow velocities are much faster with a maximum value of $0.55 \mathrm{~m} / \mathrm{s}$ and have larger longitudinal velocity component near the front face where the reaction takes place. Analysis of Qun Chen et al. [10] showed that generating longitudinal flow can enhance laminar mass transfer. As a result, the convective mass transfer in our reactor is obviously effective. Behind the deflector, the air flow even keep still, especially near the wall. At the effect of inertia force and viscous force, the flow deflects downward and vortex exists near the upper outlet.Fig.4 shows that the pressure field of the prototype reactor decreases with the deflectors' barrier. Low-pressure areas are at behind of deflectors, which may lead to eddy and increase forces acting on deflectors. The total pressure loss between inlet and outlet is $0.18 \mathrm{~Pa}$. It was obvious that the distribution of velocity and pressure of the prototype reactor need to be optimize.

\section{Structure optimization}

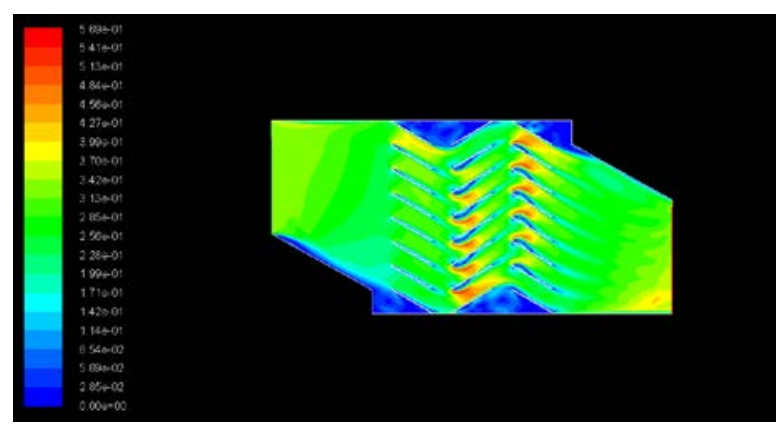

Fig.5 The velocity field of the optimized reactor with 3 modules

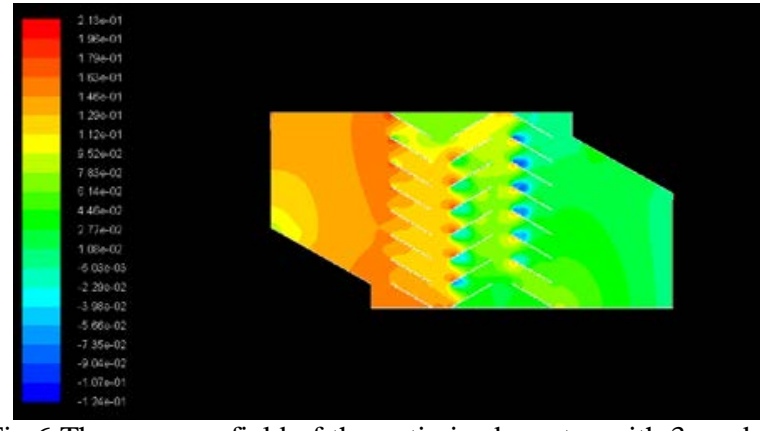

Fig.6 The pressure field of the optimized reactor with 3 modules 
With the guide of numerical analysis result, we optimal designed the inlet and outlet into contraction form. The inclination angles of inlet/outlet are equal to those of their proximal deflectors to fit the flow field. The deflectors and lamps is modular in the sense that the number of lamp-deflector modules can be increase and decrease as needed, simplify the processing and manufacturing as well. In order to study the effect of optimization, the flow field of a optimized reactor with 3 modules were simulated as the former method. The velocity at inlet reset to $0.33 \mathrm{~m} / \mathrm{s}$, since the inlet size decrease. The other conditions remained unchanged. After 240 times of iterative computation, the velocity field and pressure field were shown as Fig.5 and Fig.6. The velocity distribution becomes much more uniform. Dead zones near the wall are small, which have little influence on the contaminant removal effectiveness. The pressure lose decrease to 0.09Pa, but it is still considerable near the deflectors, so the strength of deflectors should be checked before construction.

\section{Conclusions}

A novel fixed bed photocatalytic prototype reactor with tilted photocatalyst-coated deflectors was designed and a numerical simulation for effects verification and optimization were developed by FLUENT. Promising conclusions were achieved as following.

(1) In the designed reactor, velocity distributes uniformly at the inlet and outlet, convective mass transfer is effective near the deflectors.

(2) The total pressure loss is obviously low. But the pressure lose near the deflectors are relatively high, so the strength of deflectors should be checked before construction.

(3) The modular structure simplifies the construction, improves the adaptability and expandability. The reactor can be applied widely in environmental protection industries, for instance industrial smoke dispose, engine exhaust gases purification and indoor air cleaning.

\section{Acknowledgements}

This work was financially supported by the Shanghai Science and technology Innovation Action Plan (14DZ1205300).

\section{References}

[1] J. Peral, D.F. Ollis: Catal. Vol. 136(1992), p. 554.

[2] L. Zhang, Y.H. Dong, W.D. Qi: Marine Technol. Vol. 3(2013), p. 46.

[3] F. Shiraishi, D. Ohkubo, K. Toyoda: Chemical Engineering Journal, Vol. 114(2005), p. 153.

[4] Y.X. Qian: Doctoral dissertation. Zhejiang University of Technology (2010)

[5] Guo Fei: Doctoral dissertation. Wuhan University of Technology, 2010,11.

[6] G.J. Chen: Doctoral dissertation. Nanjing University of Technology, 2004,6.

[7] Ajay K. Ray*, Antonie A.C.M. Beenackers: Catalysis Today Vol. 40 (1998) p.73.

[8] L.A. Dibble, Gregory B Raupp: Environ Sci. Technol. Vol. 26(1992), p.492.

[9] L. Zhang, P.Y. Zhang, J. Chen, China. Patent 2005100121134. (2007)

[10] Q. Chen, J.A. Meng: International Journal of Heat and Mass Transfer Vol. 51(2008), p. 2863. 